\title{
Continuously self-focusing and continuously self-defocusing two-dimensional beams in dissipative media
}

\author{
A. Ankiewicz, N. Devine, and N. Akhmediev \\ Optical Sciences Group, Research School of Physical Sciences and Engineering, Australian National University, \\ Canberra ACT 0200, Australia \\ J. M. Soto-Crespo \\ Instituto de Óptica, CSIC, Serrano 121, 28006 Madrid, Spain \\ (Received 7 January 2008; published 21 March 2008)
}

\begin{abstract}
Using the Lagrangian formalism, with a simple trial function for dissipative optical two-dimensional (2D) soliton beams, we show that there are two disjoint sets of stationary soliton solutions of the complex cubicquintic Ginzburg-Landau equation, with concave and convex phase profiles, respectively. These correspond to continuously self-focusing and continuously self-defocusing types of $2 \mathrm{D}$ solitons. Their characteristics are distinctly different, as the energy for their existence can be generated either at the center or in the outer layers of the soliton beam. These predictions are corroborated with direct numerical simulations of the GinzburgLandau equation. Regions of existence in the parameter space of these two types of solutions are found and they are in reasonable agreement with the predictions of the Lagrangian approach. In addition, direct numerical simulations allow us to find more complicated localized solutions around these regions. These solutions lack cylindrical symmetry and/or pulsate in time. Examples of the complex behavior of these beams are presented.

DOI: 10.1103/PhysRevA.77.033840

PACS number(s): 42.65.-k, 47.20.Ky
\end{abstract}

\section{INTRODUCTION}

Optical beam propagation in nonlinear media has been studied in detail by many researchers [1-5]. A number of review papers can be found in the literature on this subject $[6,7]$. In self-focusing conservative media, the main difference between the one-dimensional (1D) and twodimensional (2D) cases is the problem of collapse [8-11]. In the simple paraxial model based on the nonlinear Schrödinger equation, 2D beams are predicted to contract to an infinitely small spot with an infinite central field intensity. Various mathematical models for the description of the collapse phenomenon have been suggested $[1,2]$ and assorted mechanisms to avoid it have been proposed [3,5,11].

The situation in dissipative media of any dimensionality is quite different. Beam collapse can be arrested due to the dissipation. In complete absence of gain, losses may increase without limit as soon as the field increases to high values. On the other hand, the gain-loss balance in the medium may lead to a completely different behavior of the beam. Specifically, the energy in dissipative media is generated at some points of the beam and dissipated at others. Despite being a dynamical formation in this sense, the beam can be stationary, with both amplitude profile and phase chirp across the beam being fixed. A beam having a positive or negative chirp in a conservative nonlinear medium would necessarily change its profile on propagation. It would either self-focus or selfdefocus. Dissipative media admit a different scenario. Even with chirp, the beam may remain stationary and propagate without changing its shape.

The term "dissipative," in its present meaning, was introduced in the works of Prigogine [12] to describe systems which have losses as well as a pump source. One of the models of a dissipative system is based on the complex Ginzburg-Landau equation (CGLE) [13] that has terms re- sponsible for a variety of gain-loss mechanisms. This equation contains linear as well as nonlinear gain terms. It also describes self-phase modulation, as well as diffraction which manifests itself as a discriminator for various angular components of the beam. One of its experimental realizations is spatial dissipative solitons in semiconductors $[14,15]$. Localized solutions of the CGLE in a bulk medium are 2D beams in a three-dimensional space, with the longitudinal coordinate being the propagation direction.

In the present work, we find that there are at least two sets of stable stationary localized solutions with a major qualitative difference: One set consists of "self-focusing" beams (solitons) and the other set of "self-defocusing" beams (antisolitons [16]). When we smoothly change the parameters of the medium, viz., the external pump, nonlinearity, spectral filtering, etc., the solutions also change smoothly in a given range, but abrupt changes occur at the bifurcation points. Our studies reveal the fact that one type of soliton cannot be transformed into the other-either smoothly or through a bifurcation. In fact, they are separated in parameter space by a region where no solitons exist. There is no way of continuously transforming a soliton from one region into a soliton of the other region.

The two-dimensional beams under study in this work belong to the class of "dissipative solitons" [17]. Thus, we can make certain conclusions based on our previous experience. To start with, we consider the simplest case of stationary 2D solitons with plain "bell-shaped" profiles. This class of solutions can be well approximated with reasonably simple trial functions, and this allows us to predict the existence of two types of solutions using the method of moments or Lagrangian formalism [18]. Numerical simulations confirm the existence of these two types of beams. Moreover, numerical simulations are not restricted to cylindrically symmetric beams. Thus, they allow us to find more complicated beam 
shapes, as well as their dynamics. Such solutions do appear in the vicinity of the boundaries of existence of stationary solutions with cylindrical symmetry. In particular, we find rotating solutions of elliptic shape, as well as various complexes of several beams, and even 2D exploding beams.

\section{STATEMENT OF THE PROBLEM}

Our studies are based on an extended complex GinzburgLandau equation, that includes cubic and quintic nonlinear terms. This normalized propagation equation reads as [19]

$$
\begin{gathered}
i \psi_{z}+\frac{D}{2} \nabla_{\perp}^{2} \psi+|\psi|^{2} \psi+\nu|\psi|^{4} \psi= \\
i \delta \psi+i \epsilon|\psi|^{2} \psi+i \beta \nabla_{\perp}^{2} \psi \\
+i \mu|\psi|^{4} \psi,
\end{gathered}
$$

where $\psi(z, r)$ is the normalized envelope of the field,

$$
\nabla_{\perp}^{2}=\frac{1}{r} \frac{\partial}{\partial r}\left(r \frac{\partial}{\partial r}\right)
$$

is the transverse Laplacian for radially symmetric beams, $z$ is the propagation distance and $r$ is the radial coordinate, $D$ is the diffraction coefficient, $\nu$ is the coefficient of the quintic nonlinearity, $\delta$ represents linear losses, $\epsilon$ is the nonlinear gain coefficient, $\beta$ stands for spectral angular filtering of the cavity, and $\mu$ characterizes the saturation of the nonlinear gain. This equation is applicable to wide aperture lasers $[20,21]$ and vertical external cavity semiconductor devices [22]. It can also be applied to multimode optical fibers made of erbium doped glass, when the number of modes is very large.

There are no known analytic solutions of Eq. (1). The only way to obtain solutions is through numerical simulations. The latter requires a large amount of computer time. To have an idea of how the solution may depend on the parameters of the equation requires a vast number of simulations. We are interested in finding the regions in the space of parameters of Eq. (1) which admit stable soliton solutions. Before starting any simulations, it would be nice to have some estimates of where, in the parameter space, these solutions could be found. For that purpose, approximate techniques can be very helpful, serving as a guide for our numerical simulations. Thus, some simplification is a necessity.

The Lagrangian method [18] allows the reduction of the complete evolution problem, with an infinite number of degrees of freedom, to a much simpler one with just a few degrees of freedom. The problem of solving a partial differential equation is then reduced to solving a small set of ordinary differential equations with variables which involve the main pulse characteristics such as the peak amplitude, the pulse width, the position of its center of mass, and phase chirp parameters. For an arbitrary localized field $\psi$, a better variable than amplitude is the beam power, $Q$,

$$
Q=2 \pi \int_{0}^{\infty}|\psi|^{2} r d r,
$$

Thus, in our low-dimensional approximation, we shall describe the pulse shape by using the beam power, $Q$, the width, $f$, and a chirp factor, $c$, while locating the center of the soliton at the origin. These three nonzero quantities, together with a trial function, can give a good representation of the soliton solution.

\section{LAGRANGIAN FORMALISM AND TRIAL FUNCTION}

Let $L$ be the integral of the Lagrangian density, $L_{d}$. Hence, we have

$$
L=L_{1}+\frac{D}{2} \mathcal{P}-\frac{1}{2} S_{4}-\frac{\nu}{3} S_{6},
$$

where, we have defined

$$
S_{n}=\int_{0}^{\infty}|\psi|^{n} r d r, \quad \mathcal{P}=\int_{0}^{\infty}\left|\frac{\partial \psi}{\partial r}\right|^{2} r d r,
$$

and

$$
L_{1}=-\frac{i}{2} \int_{0}^{\infty}\left(\psi^{*} \frac{\partial \psi}{\partial z}-\psi \frac{\partial \psi^{*}}{\partial z}\right) r d r
$$

Then, $Q=2 \pi S_{2}$ is the total beam power. The rate of change of $Q$ is given by [19]

$$
Q_{z}=\frac{d Q}{d z}=4 \pi\left(\delta S_{2}-\beta P+\epsilon S_{4}+\mu S_{6}\right) .
$$

In the following, we shall use this trial function that in combination with either the minimization of the Lagrangian or by using the method of moments allow us to approximate the real solution.

\section{A. Quartic-Gaussian trial function}

In order to have more flexibility in approximating the beam shape, we use a higher-order Gaussian-type function as a trial function, viz.,

$$
\begin{aligned}
\psi(r, z)= & \frac{\sqrt{2}}{\pi^{3 / 4} f(z)} \exp \left(-\frac{r^{4}}{2 m^{2} f^{4}(z)}-\frac{r^{2}}{f^{2}(z)}\right) \\
& \times \sqrt{\frac{e^{-m^{2}} Q(z)}{m \operatorname{erfc}(m)}} \exp \left\{i\left[r^{2} c(z)-\theta(z)\right]\right\},
\end{aligned}
$$

with $f(z), c(z)$, and $Q(z)$ defined as above and where $m$ is a parameter that controls the relative contribution of each term $\left(r^{2}\right.$ and $\left.r^{4}\right)$ in the trial function. The complementary error function, $\operatorname{erfc}(m)$, appears in (4) to give the correct normalization. Throughout this paper, we consider $m$ to be a constant. The function $\theta(z)$ in (4) represents the axial phase evolution. For a stationary solution, the values $f, c$, and $Q$ are constants and $\theta^{\prime}(z)=\omega=$ const, so that the phase evolution is linear, $\theta(z)=\omega z$. For pulsating solutions with radial symmetry, $\omega=\omega(z)$ oscillates rather than being constant. Our studies have shown that the function (4) gives a good fit for the beam shape as well as for the regions of existence of solitons in the space of the equation parameters. 
Using (4) in the expression for the Lagrangian, we obtain

$$
\begin{gathered}
L_{1} \frac{2 \pi^{3 / 2}}{Q(z)}=m\left(\frac{e^{-m^{2}}}{\operatorname{erfc}(m)}-m \sqrt{\pi}\right) f^{2}(z) c^{\prime}(z)-\sqrt{\pi} \theta^{\prime}(z), \\
S_{4}=\frac{\operatorname{erfc}(\sqrt{2} m) Q^{2}(z)}{\sqrt{2} m \pi^{5 / 2} \operatorname{erfc}^{2}(m) f^{2}(z)}, \\
S_{6}=\frac{2 \operatorname{erfc}(\sqrt{3} m) Q^{3}(z)}{\sqrt{3} m^{2} \pi^{4} \operatorname{erfc}^{3}(m) f^{4}(z)} \\
\mathcal{P}=\frac{Q(z)}{\pi^{3 / 2} f^{2}(z)}\left(\frac{2 e^{-m^{2}}\left[m^{2} c^{2}(z) f^{4}(z)+1\right]}{m \operatorname{erfc}(m)}\right. \\
\left.-\sqrt{\pi}\left[2 m^{2} c^{2}(z) f^{4}(z)+1\right]\right)
\end{gathered}
$$

Euler-Lagrange equations for our dissipative system are (see [18])

$$
\frac{d}{d z}\left(\frac{\partial L}{\partial p_{z}}\right)-\frac{\partial L}{\partial p}=-2 \operatorname{Re}\left(\int_{0}^{\infty} K \frac{1}{i \psi^{*}} \frac{\partial \psi^{*}}{\partial p} r d r\right)
$$

for each parameter $p$, where $K$ indicates the dissipative terms of the CGLE, viz.,

$$
K=\delta|\psi|^{2}+\epsilon|\psi|^{4} \psi+\mu|\psi|^{6}+\beta \frac{\psi^{*}}{r} \frac{\partial}{\partial r}\left(r \psi_{r}\right) .
$$

So the Euler-Lagrange equations provide us a way of processing the trial function so that we can easily find the relevant physical quantities. This is much easier than solving the original partial differential equation (1). We can then easily find $\frac{\partial L}{\partial p}$ and $\frac{\partial L}{\partial p_{z}}$ for each $p=Q, f, c, \theta, m$.

The first three multiplicative factors needed are purely imaginary,

$$
\begin{gathered}
\frac{1}{i \psi^{*}} \frac{\partial \psi^{*}}{\partial Q}=-\frac{i}{2 Q(z)}, \\
\frac{1}{i \psi^{*}} \frac{\partial \psi^{*}}{\partial f}=-\frac{i\left(\frac{2 r^{4}}{m^{2}}+2 f^{2}(z) r^{2}-f^{4}(z)\right)}{f^{5}(z)}, \\
\frac{1}{i \psi^{*}} \frac{\partial \psi^{*}}{\partial m}=\frac{i}{2}\left(2 m+\frac{1}{m}-\frac{2 r^{4}}{m^{3} f^{4}(z)}-\frac{2 e^{-m^{2}}}{\sqrt{\pi} \operatorname{erfc}(m)}\right) .
\end{gathered}
$$

The other two are real,

$$
\begin{gathered}
\frac{1}{i \psi^{*}} \frac{\partial \psi^{*}}{\partial c}=-r^{2}, \\
\frac{1}{i \psi^{*}} \frac{\partial \psi^{*}}{\partial \theta}=1 .
\end{gathered}
$$

For example,

$$
\frac{\partial L}{\partial \theta}=-\frac{Q(z)}{2 \pi} .
$$

In fact, the $\theta$ equation recovers Eq. (3).

The trial function (4) can be improved in several ways to give a better fit for the exact solution. For example, we could use a different form for the phase term in (4). Namely, we could replace $c(z) r^{2}$ by the expression $b \ln \left[\cosh \left(\frac{r}{f(z)}\right)\right]$. This form would reflect the fact that the phase tends to be roughly quadratic near the axis $(r=0)$ and turns into a linear function when $r$ is larger. Near the center, $c \approx b / f^{2}$ and the sign of $b$ is the same as the sign of $c$. In reality, this modification does not lead to significant improvements.

Now we can either set the value of $m$, e.g., $m=$ const, and then find $Q, f, c$ or use the additional $m$ equation and solve for $Q, f, c$ and the "optimum" $m$. In practice, this latter idea leads to a more complicated dynamical system but does not give a significant improvement, so we usually study the evolution of the set of ordinary differential equations, $Q^{\prime}(z)$ $=Q_{z}, f^{\prime}(z)=f_{z}, c^{\prime}(z)=c_{z}$ [and then we find $\theta^{\prime}(z)$ ], or just get the stationary solutions from the fixed points by solving the set $Q^{\prime}(z)=0, f^{\prime}(z)=0, c^{\prime}(z)=0$, and then use $\theta^{\prime}(z)=\omega$. The fixed points are designated $Q_{0}, f_{0}, c_{0}$. Various fixed $m$ lead to different low-dimensional dynamical systems. Generally, they are complicated, with coefficients which are given in terms of hypergeometric functions of $m$. Two relatively simple cases are presented below.

\section{B. Case $m=1$}

Using Eq. (4) in the Euler-Lagrangian set of equations allow us to obtain a dynamical system for the variables $Q, f$, and $c$. For example, for the case $m=1$ we have $(D-1)$

$$
\begin{aligned}
\frac{Q_{z}}{Q(z)}= & -2.55587 \beta c^{2}(z) f^{2}(z)+2 \delta+0.93407 \frac{\epsilon Q(z)}{f^{2}(z)} \\
& -6.55587 \frac{\beta}{f^{2}(z)}+0.547538 \frac{\mu Q^{2}(z)}{f^{4}(z)}, \\
\frac{f_{z}}{f(z)}= & -0.98216 \beta c^{2}(z) f^{2}(z)+2 c(z)+2.147885 \frac{\beta}{f^{2}(z)} \\
& -0.194244 \frac{\epsilon Q(z)}{f^{2}(z)}-0.15927 \frac{\mu Q^{2}(z)}{f^{4}(z)}, \\
c_{z}= & -2 c^{2}(z)-17.0404 \frac{\beta c(z)}{f^{2}(z)}-0.730924 \frac{Q(z)}{f^{4}(z)} \\
& +\frac{5.13004958}{f^{4}(z)}-0.57127423 \frac{\nu Q^{2}(z)}{f^{6}(z)} .
\end{aligned}
$$

Approximate coefficients here have been calculated numerically. Equations (15)-(17) comprise a complete set. The phase, $\theta$, can be calculated separately from the following equation: 


$$
\begin{aligned}
\theta_{z}= & -5.44413 \beta c(z)-0.700555 \frac{Q(z)}{f^{2}(z)}+\frac{3.27793}{f^{2}(z)} \\
& -0.45628 \frac{\nu Q^{2}(z)}{f^{4}(z)} .
\end{aligned}
$$

\section{Gaussian limit}

If we take the limit $m \rightarrow \infty$, we obtain the Gaussian limit,

$$
\psi=\frac{1}{f(z)} \sqrt{\frac{2 Q(z)}{\pi}} \exp \left[-\left(\frac{r}{f(z)}\right)^{2}\right] \exp \left\{i\left[r^{2} c(z)-\theta(z)\right]\right\} .
$$

The dynamical system in this case takes the exact and simple form

$$
\begin{aligned}
& \frac{Q_{z}}{Q(z)}=-4 \beta c^{2}(z) f^{2}(z)+2 \delta+\left(\frac{2 \epsilon Q(z)}{\pi}-4 \beta\right) \frac{1}{f^{2}(z)} \\
&+\frac{8 \mu Q^{2}(z)}{3 \pi^{2} f^{4}(z)}, \\
& \frac{f_{z}}{f(z)}=-2 \beta c^{2}(z) f^{2}(z)+2 c(z)+\left(2 \beta-\frac{\epsilon Q(z)}{2 \pi}\right) \frac{1}{f^{2}(z)} \\
&-\frac{8 \mu Q^{2}(z)}{9 \pi^{2} f^{4}(z)}, \\
& c_{z}=-2 c^{2}(z)-8 \frac{\beta c(z)}{f^{2}(z)}+\left(2-\frac{Q(z)}{\pi}\right) \frac{1}{f^{4}(z)}-\frac{16 \nu Q^{2}(z)}{9 \pi^{2} f^{6}(z)},
\end{aligned}
$$

plus an additional equation for the phase

$$
\theta_{z}=-4 \beta c(z)+\left(2-\frac{3 Q(z)}{2 \pi}\right) \frac{1}{f^{2}(z)}-\frac{20 \nu Q^{2}(z)}{9 \pi^{2} f^{4}(z)} .
$$

\section{EXISTENCE OF TWO CLASSES OF BEAMS}

Stationary solutions of the dynamical system [Eqs. (15)-(17)] are given by the soliton parameters which are $z$ independent, i.e., $Q(z)=Q_{0}, f(z)=f_{0}$, and $c(z)=c_{0}$. When we have these parameters, we can use Eq. (18) to find $\theta_{z}=\omega$. Such a solution corresponds to a fixed point (FP) of the dynamical system. Fixed points of this three-variable dynamical system, together with the trial function (4), approximate the stationary solitons of the CGLE. Standard linearization techniques can be used to find the stability of these FPs. An example of the soliton profile found using the trial function of Eq. (4) is shown in Fig. 1. This particular soliton has negative chirp, $c$. The key result of the present study is that solitons can have either positive or negative chirp, $c$. They are located in different regions of the parameter space, though their profiles may be similar to each other.

Guided by the predictions of the reduced method, we have numerically calculated the exact field amplitude and phase profiles of the solutions for each region of existence of stable

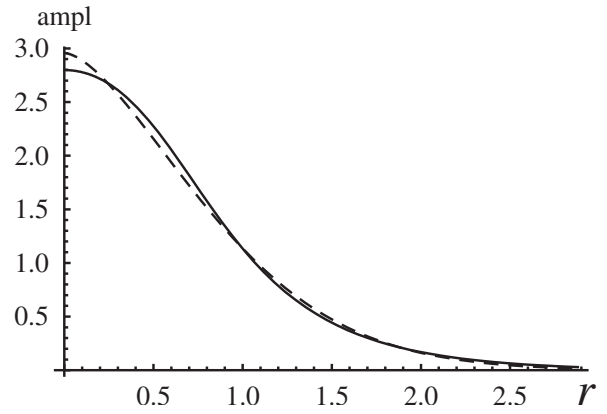

FIG. 1. An example of radial profile of a beam with negative chirp $(c<0)$. Exact profile (solid line) found using CGLE and quartic-Gaussian approximation (4) with $m=1.6$ (dashed line). Here, $\delta=-0.1, \nu=-0.08, \mu=-0.03, \epsilon=0.22, \beta=0.08$. The values $Q_{0}=13.8, f_{0}=1.03, c_{0}=-0.058$, and $\omega=-1.2$, calculated from the approximation, fit the exact result fairly well.

solitons. Direct simulations of the CGLE (1) are made without imposing radial symmetry on the beams and therefore the Laplacian depends on both variables $x$ and $y$,

$$
\nabla_{\perp}^{2}=\frac{\partial^{2}}{\partial x^{2}}+\frac{\partial^{2}}{\partial y^{2}}
$$

We have numerically solved Eq. (1) in a Cartesian grid using a split-step Fourier method. Thus, the second-order derivative terms in $x$ and $y$ are solved in Fourier space. All other linear and nonlinear terms in the equation are solved in real space using a fourth-order Runge-Kutta method. Most of the simulations presented in the paper were carried out using a numerical grid of $512 \times 512$ points in $(x, y)$. We used various values of step size along the $x, y$, and $z$ variables to check that the results do not depend on the mesh intervals, thus avoiding any numerical artifacts. In this way, we were able to find regions where stable stationary solutions exist and to determine the exact field amplitude and phase profiles of the solutions in these regions.

Generally, as expected, the beams are radially symmetric. Two examples, illustrating the two types of solitons found numerically, are shown in Figs. 2 and 3. The amplitude profiles are qualitatively similar. However, the phase profiles clearly show that the chirps are of opposite sign. Thus, these

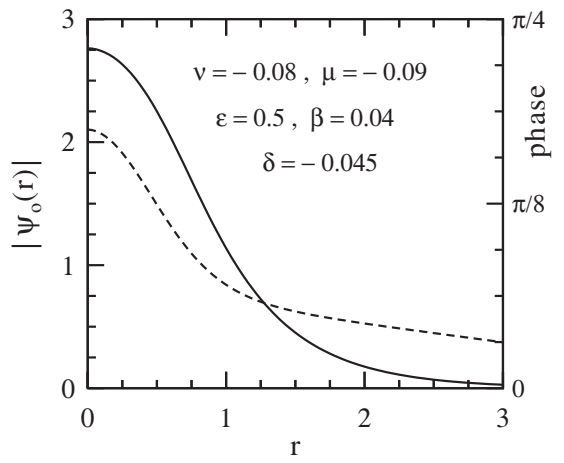

FIG. 2. Amplitude (solid line) and phase (dashed line) profiles of the soliton solution obtained for the following values of the parameters: $\beta=0.04, \nu=-0.08, \delta=-0.045, \epsilon=0.5$, and $\mu=-0.09$. 


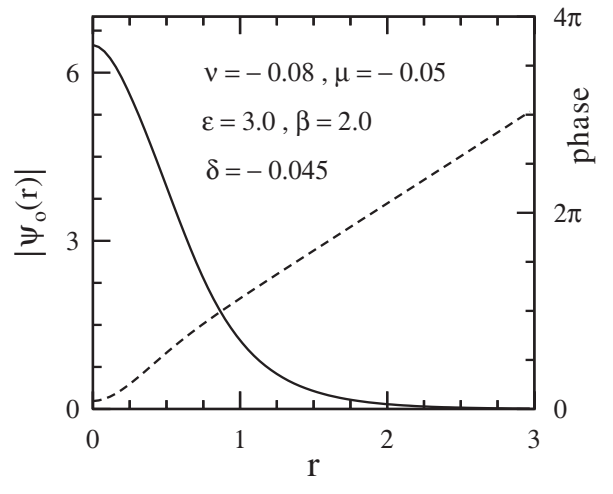

FIG. 3. Amplitude (solid line) and phase (dashed line) profiles of the antisoliton with $\epsilon=3, \beta=2$.

two examples belong to different classes of solitons. We can also notice the different phase scales in these two figuresthe positive chirp is much stronger than the negative one. The amplitude scales show that they may carry more power. These qualitative observations are in full agreement with the predictions of the low-dimensional approximation.

\section{TWO REGIONS OF EXISTENCE}

By solving either of the dynamical systems for various values of the equation parameters of the CGLE, we can construct regions of stable fixed points. In order to keep the presentation of the results reasonably simple, we keep four equation parameters fixed and change the other two, looking at these two-dimensional slices of the six-dimensional space of the equation parameters $(D, \beta, \epsilon, \delta, \mu$, and $\nu)$ to find where FPs exist and are stable. Proceeding in this way, we have observed the existence of two separate regions of stable fixed points. A point from one region cannot be transformed into a point from the other region with a continuous change of parameters. Thus, it appears that these two regions correspond to two different types of solitons of the CGLE. One of the branches has positive chirp, $c$, and high power, $Q$, while the other one has negative chirp and low power. Within the low-dimensional approximation, FPs in both regions are stable.

Figures 4 and 5, respectively, show two 2D regions where these two types of different solutions exist. In particular, Fig. 4 shows the region of existence of solitons with negative chirp. The negative chirp means that the beam is continuously self-focusing. Figure 5 shows the region of existence of solitons with positive chirp. Such a beam is continuously self-defocusing. The gray area in each case represents the results obtained from the low-dimensional approach, while the hatched area is obtained from numerical simulations of the whole CGLE, as described above. The region obtained with the reduced model approximates the exact results quite well in the case of self-focusing solitons. The agreement is qualitatively reasonable for the region of self-defocusing beams (antisolitons). A similar situation has been observed in the $1 \mathrm{D}$ case [16].

Thus, using the low-dimensional approximation, we predict the existence of two types of $2 \mathrm{D}$ beams with qualita-

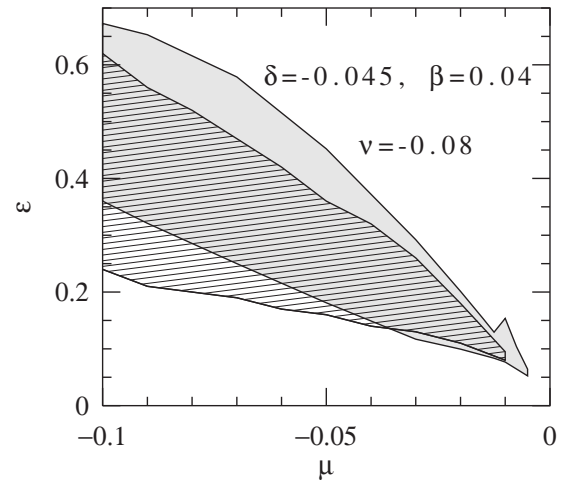

FIG. 4. Region of existence of solitons with negative chirp $\left(c_{0}\right.$ $<0)$ in the $(\mu, \epsilon)$ plane found using the trial function (4). Stable FPs exist in the gray region. The hatched area shows the region where stable stationary solutions are obtained numerically using the full Ginzburg-Landau equation. The two regions match reasonably well.

tively different properties. The beams of one class have negative chirp and are continuously self-focusing, while beams of the other type have positive chirp and are continuously self-defocusing. However, each type of beam remains stationary along the direction of propagation, $z$. These two types of beams are located in two different regions of the parameter space and are not connected to each other. Continuously self-defocusing beams normally have higher power, $Q$, than continuously self-focusing ones.

\section{SOLITON CHIRP AND ENERGY FLOW ACROSS THE SOLITONS}

The soliton chirp is an important physical parameter that is related to the energy flow inside the beam. In order to see this, let us recall the continuity equation for dissipative systems [17]. For stationary solutions, the density of energy generation, $\hat{P}(r)$ is related to $\hat{j}(r)$ through the following equation:

$$
\hat{P}(r)=\frac{1}{r} \frac{\partial}{\partial r}(r \hat{j}),
$$

where

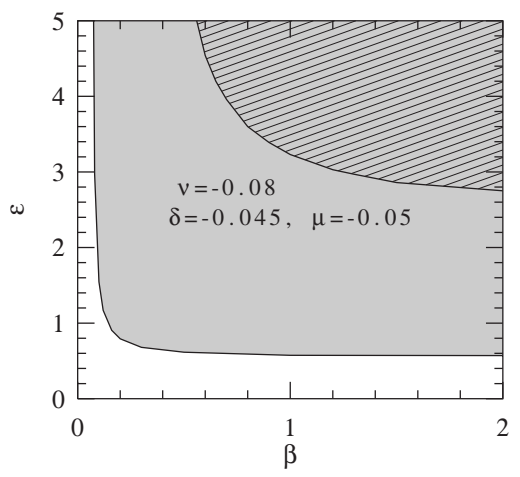

FIG. 5. Region of existence of antisolitons with positive chirp $\left(c_{0}>0\right)$ (or self-defocusing type of solitons) in the $(\beta, \epsilon)$ plane. The gray region is obtained with the low-dimensional approximation while the hatched region is obtained with direct numerical simulations. The parameters are $D=1, \nu=-0.08, \mu=-0.05, \delta=-0.045$. 


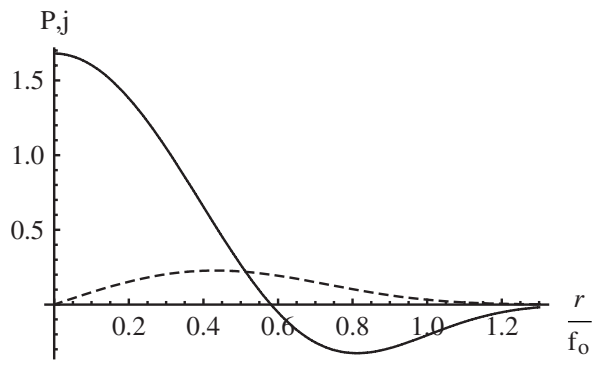

FIG. 6. Normalized energy generation (solid line) and flux (dashed line) as a function of the normalized radius $R=r / f_{0}$. Here, $m=1$.

$$
\hat{j}=\frac{i}{2}\left(\psi \psi_{r}^{*}-\psi_{r} \psi^{*}\right)
$$

and

$$
\begin{aligned}
\hat{P}(r)= & 2 \delta|\psi|^{2}+2 \epsilon|\psi|^{4}+2 \mu|\psi|^{6} \\
& +\beta\left(|\psi|_{r r}^{2}-2\left|\psi_{r}\right|^{2}+\frac{1}{r} \frac{\partial}{\partial r}|\psi|^{2}\right) .
\end{aligned}
$$

Now, we can use the reduced model to relate the chirp to the energy generation and flux across the beam.

Using the trial function (4) for arbitrary $m$, we find the flux to be

$$
\hat{j}=\frac{4 R c_{0} Q_{0}}{m \pi^{3 / 2} \operatorname{erfc}(m) f_{0}} \exp \left(-\frac{\left(m^{2}+R^{2}\right)^{2}}{m^{2}}\right),
$$

where $R=r / f_{0}$. Then the energy generation $\hat{P}$ is given by

$$
\hat{P}(R)=\frac{8\left[m^{2}\left(1-2 R^{2}\right)-2 R^{4}\right] c_{0} Q_{0}}{m^{3} \pi^{3 / 2} \operatorname{erfc}(m) f_{0}^{2}} \exp \left(-\frac{\left(m^{2}+R^{2}\right)^{2}}{m^{2}}\right) .
$$

Normalized values of $P(R)=\hat{P} \frac{f_{0}^{2}}{c_{0} Q_{0}}$ and $j(R)=\hat{j} \frac{f_{0}}{c_{0} Q_{0}}$ are plotted in Fig. 6. These values do not depend on the chirp. $P(R)$ is positive at the center of the beam while $j(R)$ is positive in the whole interval.

We have the condition that the total energy generation is zero:

$$
\int_{0}^{\infty} R P(R) d R=0
$$

This condition must be satisfied for stationary solutions, and is clearly valid here for all $m$. As we can see from Eqs. (27) and (28), the values $\hat{j}$ and $\hat{P}$ are directly proportional to $c_{0}$. If $c_{0}<0$, energy is generated in the outer parts of the beam where $\hat{P}>0$, and flows to the central area where it is dissipated. In a conservative medium, this process would correspond to the beam self-focusing. If $c_{0}>0$, then the process is the opposite: The energy is generated near to the central axis where $\hat{P}>0$, and it is dissipated in the outer parts of the beam where $\hat{P}<0$. Again, in a conservative medium, this would correspond to the self-defocusing of the beam.

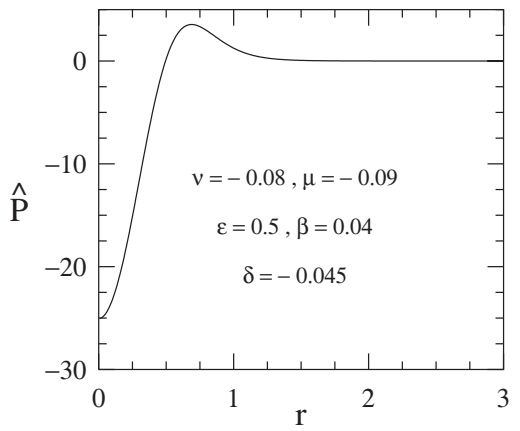

FIG. 7. Energy generation as function of the radius $r$ for the self-focusing soliton shown in Fig. 2.

Clearly, in the case of a conservative medium, the beam could not be stationary when either self-focusing or selfdefocusing occurs. In dissipative media, this effect, involving an internal radial flux of energy in the beam, produces the dynamical equilibrium within the stationary solution. Thus, the beam can be either continuously self-focusing or continuously self-defocusing.

We could use a different form for the phase term, viz., $b \ln \left[\cosh \left(\frac{r}{f(z)}\right)\right]$ to replace $c(z) r^{2}$ in Eq. (4). As mentioned above, this form reflects the fact that the phase tends to be roughly quadratic near the axis $(r=0)$ and linear when $r$ is larger. We then obtain, for the flux,

$$
\hat{j}=\frac{2 \tanh (R) b Q_{0}}{m \pi^{3 / 2} \operatorname{erfc}(m) f_{0}^{3}} \exp \left(-\frac{\left(m^{2}+R^{2}\right)^{2}}{m^{2}}\right) .
$$

For the energy generation, we obtain the following expression:

$$
\hat{P}=\frac{P_{1} b Q_{0}}{m^{3} \pi^{3 / 2} R \operatorname{erfc}(m) f_{0}^{4}} \exp \left(-\frac{\left(m^{2}+R^{2}\right)^{2}}{m^{2}}\right) .
$$

where

$$
P_{1}=2 m^{2} R \operatorname{sech}^{2}(R)+2\left[m^{2}\left(1-4 R^{2}\right)-4 R^{4}\right] \tanh (R) .
$$

The curves for normalized $j$ and $P$ would be similar to those in Fig. 6, as $b \ln [\cosh (R)] \approx R^{2} / 2$ for small $R$.

The different signs of chirp for the two types of solitons mean that, in one case, the on-axis phase is retarded relative to the outer part (negative $c$ or $b$ ), while in the other case it is advanced with respect to the outer part (positive $c$ or $b$ ).

Turning now to the exact results, we plot the distribution of energy, $P$, generated and dissipated inside of the soliton, as found numerically, directly from the CGLE. These are shown in Figs. 7 and 8. In the first case, energy is generated in the outer parts of the soliton and dissipated near the center of the beam (see Fig. 7), while in the second case, the energy generation is positive near the axis of the soliton and negative in the wings (see Fig. 8). Thus, there is a fundamental qualitative difference between the two types of solitons. We can also designate them "dissipative solitons" (negative $c$ or $b$ ) and "dissipative antisolitons" (positive $c$ or $b$ ), in analogy with the $1 \mathrm{D}$ case [16]. Generally, antisolitons tend to have high positive chirp and higher power $Q$ than negatively chirped solitons. 


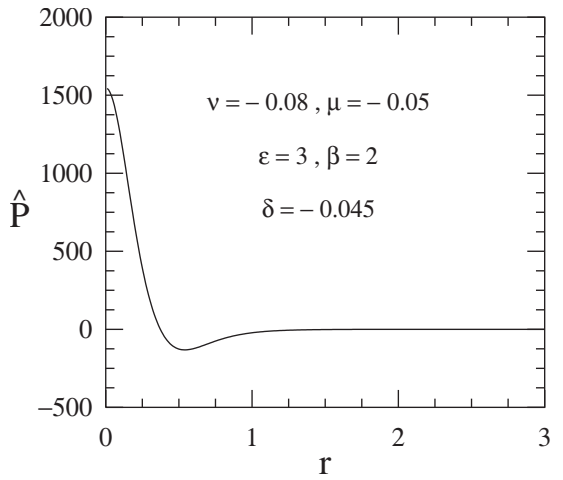

FIG. 8. Energy generation as function of the radius $r$ for the self-defocusing soliton shown in Fig. 3.

In 2D geometry, the first case corresponds to continuously self-focusing solitons, while the second one corresponds to continuously self-defocusing solitons. In each case, the beam remains stationary, i.e., it does not change its transverse profile. This shows a conceptual difference between beam propagation in conservative and dissipative media.

\section{WHAT HAPPENS AROUND THE AREAS WHERE STATIONARY SOLUTIONS BECOME UNSTABLE?}

When the radially symmetric solution loses stability, it may be transformed into a more complicated beam. Direct simulations reveal that, in addition to stationary beams, there are localized structures that cannot easily be obtained using simple approximations. The regions of existence of regular solitons or antisolitons are surrounded by regions of nonstationary beams. A rich variety of such solutions exists, with unique dynamics for each type.

Above the region of soliton beams (Fig. 4), and partially overlapping with it, we find regions that correspond to stable rotating beam complexes. These regions are shown in Fig. 9. The type of solution that we obtain depends on the equation parameters, as well as on the initial conditions, since several stable solutions exist for the same set of parameters. We observe three major types of rotating structures. The regions

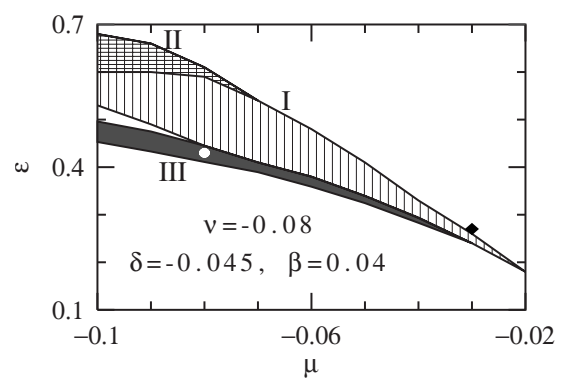

FIG. 9. Regions of existence of stable rotating structures. These regions are located around (mainly above) the upper part of the hatched region in Fig. 4. Here the vertically hatched area (denoted I) corresponds to rotating double beams. The horizontally hatched area (denoted II) corresponds to oscillating and simultaneously rotating double beams. The dark area (denoted III) corresponds to rotating oval-shaped single beams.

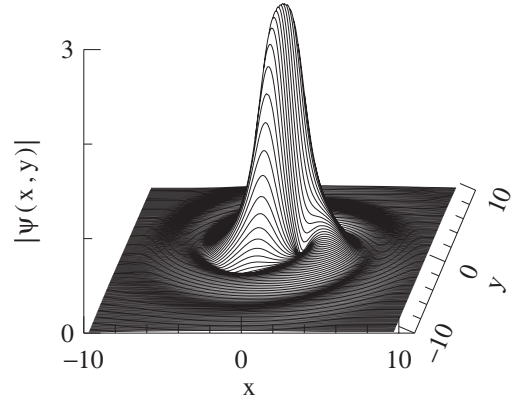

FIG. 10. Transverse profile of a rotating soliton existing in the dark region of Fig. 9. Its exact location in the parameter space in region III is marked in Fig. 9 by a white circle.

of their existence can slightly overlap with each other, indicating bistability at the borders between different regions. Thus, small hysteresis loops can be observed when increasing or decreasing the $\epsilon$ value.

In particular, some of the observed structures consist of two or more beams with circular symmetry, combined into a single beam with higher energy. Similar structures were found previously in the three-dimensional case [23] for the transversal profiles. Relatively simple double-peaked beams, lacking circular symmetry, and rotating with constant angular velocity, are observed in region I of Fig. 9. When we change the parameters and reach region II, the distance between the two beams forming the complex oscillates. In the first case (from region I to region II), the power, $Q$ is constant, while in the second case (region II), it oscillates. Starting from a solution in region I and decreasing $\epsilon$, the separation between the two components of the complex decreases, reaching a point at which the two beams merge and a beam of oval shape appears. It rotates with constant angular velocity. An example is shown in Fig. 10. However, this scenario does not always occur-for smaller values of $\mu$ (below -0.08), the regions I and III separate (note the white stripe at the smallest values of $\mu$ in Fig. 9). Then we cannot transform one solution into another by smoothly changing $\epsilon$.

Comparing Figs. 4 and 9, we can see that cylindrically symmetric beams co-exist with rotating solutions in region III and also partially in region II, resulting in bistability in a relatively wide region.

To summarize, Fig. 9 shows three distinctive regions. The vertically hatched region I corresponds to stable rotating double-beam structures. The horizontally hatched region II corresponds to periodic double soliton structures with oscillating separation between the two beams. The dark gray region III corresponds to rotating oval-shaped single beams. An example of such a beam is plotted in Fig. 10. Its location in region III of Fig. 9 is denoted by a white circle. The upper boundary of region I in Fig. 9 has additional substructures which cannot be resolved on the scale of this figure. Specifically, the beams tend to become wider, and complexes of several solitons can be observed. One example is shown in Fig. 11. This solution appears at the point denoted by the dark rhombus in Fig. 9. 


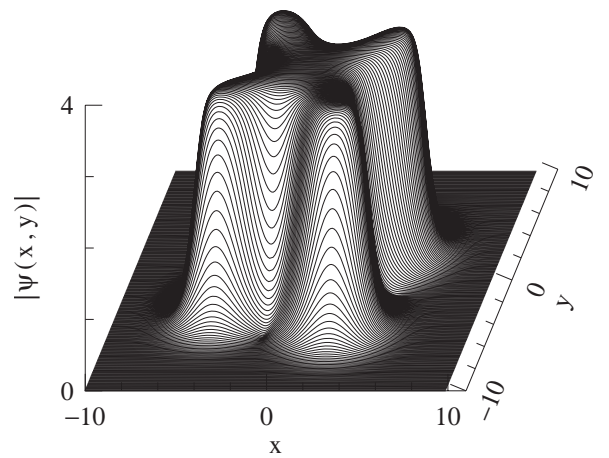

FIG. 11. Purely rotating quadruple beam. Its location in the parameter space is represented by the dark rhombus in Fig. 9.

\section{NONSTATIONARY STRUCTURES AROUND ANTISOLITONS}

The region of existence of antisolitons in Fig. 5 is also surrounded by regions of nonstationary structures that can be found using direct numerical simulations of the CGLE. These solutions are qualitatively different from those described in the preceding section. Figure 12 presents a magnification of part of the hatched region shown in Fig. 5. In addition to the hatched region, it shows, in gray, a region where soliton solutions evolve periodically. One example of radially symmetric soliton pulsations is shown in Fig. 13. The solution conserves the circular symmetry on propagation, but its profile changes periodically between the two shapes represented in parts (a) and (b) of Fig. 13. The total power $Q$ has extrema at these points. Their locations, in $z$, are indicated by the dashed lines in Fig. 13(c).

The antisolitons have higher powers than solitons, and correspondingly, they can be easily transformed into exploding solitons. For the $1 \mathrm{D}$ case, they were discovered in $[24,25]$. Figure 14 gives the evolution of power $Q$ in $z$ for an exploding soliton existing in the $2 \mathrm{D}$ case. These solutions are found in the upper left-hand side of the region for antisolitons (not shown here). The soliton explodes intermittently, thus resulting in significant bursts of power above the average, recovering the initial radially cylindrical shape after each explosion. As it was shown for the 1D exploding solitons, this unusual dynamics appears as a result of an insta-

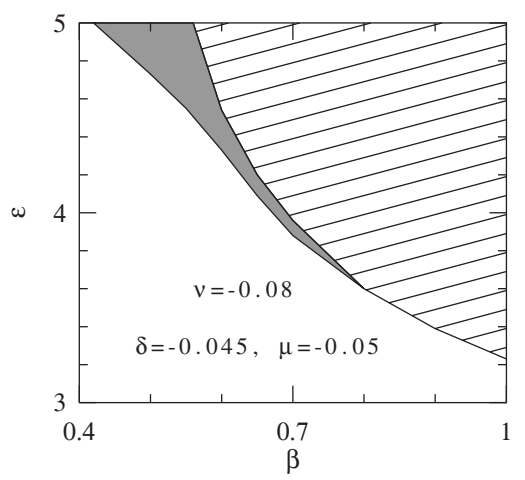

FIG. 12. Region of existence of stable antisolitons (hatched area). This is a magnification of a part of Fig. 5 The gray area represents the region of pulsating solutions.
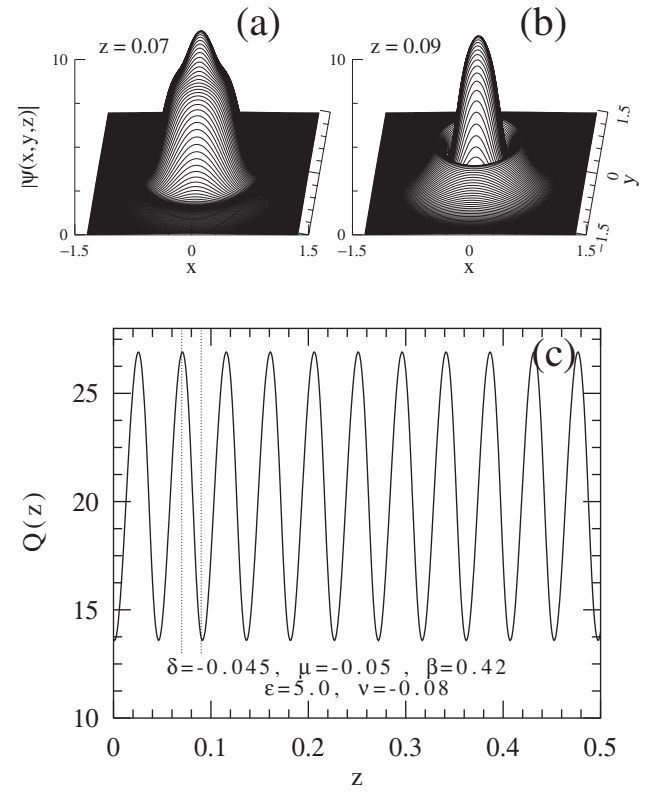

FIG. 13. Example of pulsating solution. Beam profile of the solution at (a) a maximum and (b) a minimum of $Q$. (c) Periodic evolution of $Q$ vs $z$.

bility. They can be clearly observed in the manner described above close to the left-hand side of the gray area in Fig. 12. As we move further from this boundary, the explosions become more violent, and, as a result more than one beam can be generated in some cases. This gives rise to a very complicated dynamics, ending up with the whole numerical grid filled with the solution. These type of solitons need more numerical studies to be understood in their full complexity.

\section{CONCLUSION}

In conclusion, using the Lagrangian formalism with a quartic-Gaussian trial function for dissipative optical 2D soliton beams, we have shown that there are two sets of stationary soliton solutions of the complex cubic-quintic Ginzburg-Landau equation. Their properties are distinctly different. First, the parameter regions of existence of these solitons are well separated. Second, these two types of solitons have opposite signs of the chirp parameter which gov-

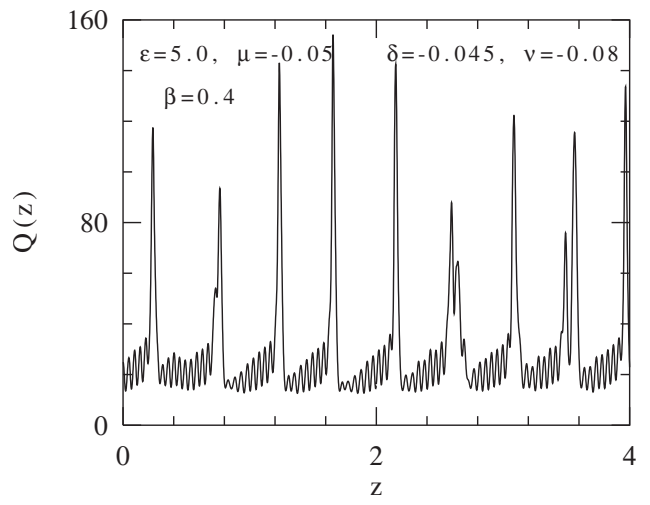

FIG. 14. Evolution of the power for an exploding beam. 
erns the direction of energy flow inside the soliton beam. As a consequence, they have concave and convex phase profiles, respectively. Thus, the beams of one type are continuously self-focusing, while the beams of the other type are continuously self-defocusing 2D solitons. This type of beam evolution can only happen in dissipative media with gain and loss. We have labeled them dissipative solitons and antisolitons, respectively.

Our predictions are corroborated by direct numerical simulations of the Ginzburg-Landau equation. Regions of existence in the parameter space of these two types of solutions are in reasonable agreement with the predictions of the Lagrangian approach.

Direct numerical simulations also reveal more complicated localized solutions around these regions. In particular, around the boundaries of existence of these two types of stationary solutions, we have observed rotating and/or oscillating beams with complex shapes.
These solutions lack radial symmetry and can have oval shape. Moreover, they can consist of two or four beams of basic structure. The solutions may rotate as well. These solutions appear close to the upper boundary of existence of regular solitons. Another type of dynamical behavior is provided by pulsating solitons. Pulsating solutions are circularly symmetric and they can be transformed into exploding beams when changing equation parameters. The latter are observed at the boundary of existence of antisolitons. We have presented numerical examples of the complex behavior of these beams. The latter type of soliton beams cannot easily be predicted using a reduced model.

\section{ACKNOWLEDGMENTS}

This research was supported under Australian Research Council's Discovery Projects funding scheme (Contract No. DP0663216). The work of one of the authors (J.M.S-C.) was supported by the MEyC under Contract No. FIS2006-03376.
[1] V. Zakharov and V. Synakh, Zh. Eksp. Teor. Fiz. 68, 940 (1975) [Sov. Phys. JETP 41, 465 (1975)].

[2] V. N. Malkin, Phys. Lett. A 151, 285 (1990).

[3] N. Akhmediev, A. Ankiewicz, and J. M. Soto-Crespo, Opt. Lett. 18, 411 (1993).

[4] E. A. Kuznetsov, J. Juul Rasmussen, K. Ripdal, and S. K. Turitsyn, Physica D 87, 273 (1995).

[5] G. Fibich, Phys. Rev. Lett. 76, 4356 (1996).

[6] J. J. Rasmussen and K. Ripdal, Phys. Scr. 33, 481 (1986).

[7] L. Berge, Phys. Rep. 303, 260 (1998).

[8] M. D. Feit and J. A. Fleck, J. Opt. Soc. Am. B 5, 633 (1988)

[9] J. T. Manassah and B. Gross, Opt. Lett. 17, 976 (1992).

[10] V. E. Zakharov, Zh. Eksp. Teor. Fiz. 62, 1747 (1972) [Sov. Phys. JETP 35, 908 (1972)].

[11] G. G. Luther, A. C. Newell, and J. V. Moloney, Physica D 74, 59 (1994).

[12] G. Nicolis and I. Prigogine, Self Organization in Nonequilibrium Systems: From Dissipative Structures to Order Through Fluctuations (Wiley, New York, 1977).

[13] I. S. Aranson and L. Kramer, Rev. Mod. Phys. 74, 99 (2002).

[14] E. A. Ultanir, G. I. Stegeman, D. Michaelis, C. H. Lange, and F. Lederer, Phys. Rev. Lett. 90, 253903 (2003).

[15] E. A. Ultanir, G. I. Stegeman, C. H. Lange, and F. Lederer, Opt. Lett. 29, 283 (2004).
[16] A. Ankiewicz, N. Devine, N. Akhmediev, and J. M. SotoCrespo, Phys. Lett. A 370, 454 (2007).

[17] N. Akhmediev and A. Ankiewicz, "Dissipative solitons in the complex Ginzburg-Landau and Swift-Hohenberg equations," in Dissipative Solitons, edited by N. Akhmediev and A. Ankiewicz (Springer-Verlag, Berlin, 2005).

[18] A. Ankiewicz, N. Devine, and N. Akhmediev, Opt. Fiber Technol. 13, 91 (2007).

[19] See, e.g., N. Akhmediev and A. Ankiewicz, Solitons: Nonlinear Pulses and Beams (Chapman and Hall, London, 1997), Chap. 11.

[20] R. Meucci, A. Labate, M. Ciofini, and Peng-Ye Wang, Quantum Semiclassic. Opt. 10, 803 (1998).

[21] S. V. Fedorov, N. N. Rosanov, A. N. Shatsev, N. A. Veretenov, and A. G. Vladimirov, IEEE J. Quantum Electron. 39, 197 (2003).

[22] J.-F. Mercier and J. V. Moloney, Phys. Rev. E 66, 036221 (2002).

[23] J. M. Soto-Crespo, N. Akhmediev, and Ph. Grelu, Phys. Rev. E 74, 046612 (2006).

[24] J. M. Soto-Crespo, N. Akhmediev, and A. Ankiewicz, Phys. Rev. Lett. 85, 2937 (2000).

[25] N. Akhmediev, J. M. Soto-Crespo, and G. Town, Phys. Rev. E 63, 056602 (2001). 